\title{
COMPLETELY METRIZABLE SPACES OF EMBEDDINGS
}

\author{
R. A. MCCOY
}

\begin{abstract}
A study is made of the complete metrizability of spaces of embeddings and spaces of homeomorphisms under the compact-open topology. It is shown that the space of continuous injections from a hemicompact metric space into a complete metric space is completely metrizable.
\end{abstract}

If $X$ is a hemicompact $k$-space and $Y$ is completely metrizable, then the space $C(X, Y)$ of continuous functions from $X$ into $Y$ with the compact-open topology is completely metrizable (see [2] and [3]). The converse is also true if $Y$ contains an arc. But complete metrizability is not a hereditary property, so that certain natural subspaces of $C(X, Y)$, such as the space $E(X, Y)$ of embeddings of $X$ into $Y$, may not be completely metrizable whenever $C(X, Y)$ is completely metrizable. Even the space $H(X)$ of homeomorphisms from $X$ into itself may not be completely metrizable whenever $X$ is a hemicompact complete metric space. The following theorem shows however that the space $I(X, Y)$ of continuous injection is in fact completely metrizable assuming reasonable properties of $X$ and $Y$. All function spaces will have the compact-open topology.

THEOREM. If $X$ is a hemicompact metric space and $Y$ is a complete metric space, then $I(X, Y)$ is completely metrizable.

Proof. Let us first assume that $X$ is compact. Since $Y$ is a complete metric space, the supremum metric $d$ on $C(X, Y)$ is a compatible complete metric. Therefore it suffices to show that $I(X, Y)$ is a $G_{\delta}$-set in $C(X, Y)$. To this end, for each $\phi$ in the space of real-valued continuous functions on $X, C(X)$, and for each $\varepsilon>0$, define

$$
G(\phi, \varepsilon)=\{f \in C(X, Y) \mid d(\phi, \psi \circ f)<\varepsilon \text { for some } \psi \in C(Y)\} .
$$

To see that each $G(\phi, \varepsilon)$ is open in $C(X, Y)$, let $f \in G(\phi, \varepsilon)$. Then $d(\phi, \psi \circ f)<\varepsilon$ for some $\psi \in C(Y)$; let $\delta=\varepsilon-d(\phi, \psi \circ f)$. Since $f(X)$ is compact, there exists a Lebesgue number $\lambda$ for $\left\{\psi^{-1}(B(r, \delta / 2)) \mid r \in \mathbf{R}\right\}$ as an open cover of $f(X)$, where $B(r, \delta / 2)$ stands for the open ball of radius $\delta / 2$ centered at $r$. Let $g \in B(f, \lambda)$, and let $x \in X$. Then $|\psi(f(x))-\psi(g(x))|<\delta$, so that

$$
\begin{aligned}
|\phi(x)-\psi(g(x))| & \leqslant|\phi(x)-\psi(f(x))|+|\psi(f(x))-\psi(g(x))| \\
& <d(\phi, \psi \circ f)+\delta=\varepsilon .
\end{aligned}
$$

Therefore $B(f, \lambda) \subseteq G(\phi, \varepsilon)$, making $G(\phi, \varepsilon)$ open in $C(X, Y)$.

Received by the editors May 20, 1981 and, in revised form, July 16, 1981.

1980 Mathematics Subject Classification. Primary 54C35; Secondary 54C25, 54E50.

Key words and phrases. Spaces of embeddings, spaces of homeomorphisms, complete metrizability, first category. 
Let $\left\{\phi_{n} \mid n \in \mathbf{N}\right\}$ be a countable dense subset of $C(X)$, which exists since $X$ is a separable metric space. To see that

$$
I(X, Y)=\cap\left\{G\left(\phi_{n}, 1 / m\right) \mid m, n \in \mathbf{N}\right\},
$$

let $h \in I(X, Y)$, let $\phi \in C(X)$, and let $\varepsilon>0$. Since $h(X)$ is closed in $Y, \phi \circ h^{-1}$ : $h(X) \rightarrow \mathbf{R}$ has a continuous extension, $\psi$, to all of $Y$. Then $\psi \circ h=\phi$, so that $h \in G(\phi, \varepsilon)$. Therefore $I(X, Y) \subseteq G(\phi, \varepsilon)$ for every $\phi \in C(X)$ and $\varepsilon>0$. To establish containment in the other direction, let $f \in \cap\left\{G\left(\phi_{n}, 1 / m\right) \mid m, n \in N\right\}$. We must show that $f$ is one-to-one, so let $x$ and $y$ be distinct elements of $X$. Since $\left\{\phi_{n} \mid n \in \mathbf{N}\right\}$ is dense in $C(X)$, there exists an $n$ such that $\phi_{n}(x) \neq \phi_{n}(y)$. Now choose $m \in \mathbf{N}$ such that $2 / m<\left|\phi_{n}(x)-\phi_{n}(y)\right|$. If $f(x)$ were equal to $f(y)$, then for every $\psi \in C(Y)$,

$$
\begin{aligned}
2 / m & <\left|\phi_{n}(x)-\phi_{n}(y)\right| \\
& \leqslant\left|\phi_{n}(x)-\psi(f(x))\right|+|\psi(f(x))-\psi(f(y))|+\left|\psi(f(y))-\phi_{n}(y)\right| \\
& =\left|\phi_{n}(x)-\psi(f(x))\right|+\left|\phi_{n}(y)-\psi(f(y))\right| \\
& \leqslant 2 d\left(\phi_{n}, \psi \circ f\right) .
\end{aligned}
$$

But this says that $d\left(\phi_{n}, \psi \circ f\right)>1 / m$ for every $\psi \in C(Y)$, which contradicts $f$ being in $G\left(\phi_{n}, 1 / m\right)$. Therefore $f(x) \neq f(y)$, so that $f$ is in $I(X, Y)$.

We now consider the more general case that $X$ is hemicompact. Then we may write $X=\cup\left\{A_{n} \mid n \in \mathrm{N}\right\}$, where each $A_{n}$ is a compact subset of $X$, each $A_{n}$ is contained in the interior of $A_{n+1}$, and each compact subset of $X$ is contained in some $A_{n}$. Then by the previous part of the proof, $\Pi\left\{I\left(A_{n}, Y\right) \mid n \in \mathbf{N}\right\}$ is completely metrizable. It will suffice to embed $I(X, Y)$ as a closed subspace of $\Pi\left\{I\left(A_{n}, Y\right) \mid n\right.$ $\in \mathbf{N}\}$.

Let $\phi$ be the natural projection from the disjoint sum $\Sigma\left\{A_{n} \mid n \in \mathbf{N}\right\}$ onto $X$. Since this is a continuous compact-covering map, the induced function

$$
\phi^{*}: C\left(\sum_{n} A_{n}, Y\right) \rightarrow C(X, Y)
$$

defined by $\phi^{*}(f)=f \circ \phi$, is an embedding. Now there is a natural homeomorphism $\alpha$ from $C\left(\sum_{n} A_{n}, Y\right)$ onto $\Pi_{n} C\left(A_{n}, Y\right)$. If $i$ is the inclusion map from $I(X, Y)$ into $C(X, Y)$, then define

$$
\Phi: I(X, Y) \rightarrow \prod_{n} C\left(A_{n}, Y\right)
$$

by $\Phi=\alpha \circ \phi^{*} \circ i$, which is an embedding. Let $Z$ be the image of $\Phi$. It should be clear that $Z$ is actually contained in $\Pi_{n} I\left(A_{n}, Y\right)$.

To see that $Z$ is closed in $\Pi_{n} I\left(A_{n}, Y\right)$, let $\left\langle f_{n}\right\rangle \in\left(\Pi_{n} I\left(A_{n}, Y\right)\right)-Z$. Suppose that for every $n,\left.f_{n+1}\right|_{A_{n}}=f_{n}$. Then since each $A_{n} \subseteq$ int $A_{n+1},\left\{\left.f_{n}\right|_{\text {int } A_{n}} \mid n \in \mathbf{N}\right\}$ is a compatible family of continuous functions whose domains form an open cover of $X$. Therefore the combination function $f$ is continuous, and will also be one-to-one. But then $f \in I(X, Y)$, while $\Phi(f)=\left\langle f_{n}\right\rangle$, which is a contradiction. Therefore there exists an $n \in \mathrm{N}$ and an $x \in A_{n}$ such that $f_{n+1}(x) \neq f_{n}(x)$. Let $V$ and $W$ be disjoint 
open subsets of $Y$ containing $f_{n}(x)$ and $f_{n+1}(x)$, respectively. Then if $[[x, V]]$ denotes the set of functions taking $x$ into $V$,

$$
\left\langle f_{n}\right\rangle \in \pi^{-1}([[x, V]]) \cap \pi_{n+1}^{-1}([[x, W]]) \subseteq\left(\prod_{n} I\left(A_{n}, Y\right)\right)-Z .
$$

Since the space $E(X, Y)$ of embeddings of $X$ into $Y$ is equal to $I(X, Y)$ when $X$ is compact, then we have the following special case (which can be found in [4, §5.1]).

COROllary 1. If $X$ is a compact metric space and $Y$ is a complete metric space, then $E(X, Y)$ is completely metrizable.

Also since the space $H(X)$ of homeomorphisms from $X$ onto itself is a closed subspace of $E(X, X)$ when $X$ is compact, then we obtain a result about $H(X)$.

COROllaRY 2. If $X$ is a compact metric space, then $H(X)$ is a completely metrizable topological group.

However, whenever $X$ is hemicompact rather than compact, Corollaries 1 and 2 are false, as we shall see in Examples 3-5. But first we look at some nice examples for contrast.

EXAMPLE 1. For each $n$, the space $E\left(\mathbf{R}^{n}, \mathbf{R}^{n}\right)$ is equal to $I\left(\mathbf{R}^{n}, \mathbf{R}^{n}\right)$, and is therefore completely metrizable.

Proof. Let $f \in I\left(\mathbf{R}^{n}, \mathbf{R}^{n}\right)$. For each $m \in \mathbf{N}$, let $A_{m}$ be the closed ball in $\mathbf{R}^{n}$ of radius $m$ centered at the origin. Then for each $m,\left.f\right|_{A_{m}}$ is an embedding. Because of invariance of domain, $f\left(\right.$ int $\left.A_{m}\right)$ is open in $\mathbf{R}^{n}$ and hence open in $f\left(\mathbf{R}^{n}\right)$.

EXAMPLE 2. For each $n$, the space $H\left(\mathbf{R}^{n}\right)$ is a $G_{\delta}$-set in $E\left(\mathbf{R}^{n}, \mathbf{R}^{n}\right)$, and is therefore completely metrizable.

Proof. For each $n$, define

$$
G_{n}=\left\{f \in E\left(\mathbf{R}^{n}, \mathbf{R}^{n}\right) \mid A_{n} \subseteq f\left(\mathbf{R}^{n}\right)\right\},
$$

where each $A_{n}$ is the closed ball in $\mathbf{R}^{n}$ of radius $n$ centered at the origin. It is clear that $H\left(\mathbf{R}^{n}\right)=\cap\left\{G_{n} \mid n \in \mathbf{N}\right\}$. To see that each $G_{n}$ is open, let $f \in G_{n}$. Then $f^{-1}\left(A_{n}\right)$ is compact, so that there exists an $m \in \mathbf{N}$ such that $f^{-1}\left(A_{n}\right) \subseteq$ int $A_{m}$. Now [[ $A_{m+1}-$ int $\left.\left.A_{m}, \mathbf{R}^{n}-A_{n}\right]\right]$ is a basic neighborhood of $f$ which, because of properties of $\mathbf{R}^{n}$, must be contained in $G_{n}$.

In order to obtain some examples which are not completely metrizable, it will suffice to find examples which are of first category in themselves. So in the following lemma we establish a condition which will help us obtain first category function spaces.

LeMma. Let $X$ be a locally compact space, and let $(Y, d)$ be a metric space. Let $F$ be a subspace of $E(X, Y)$ having the following property: there exists an $x_{0} \in X$ such that for every $\varepsilon>0$, for every compact $A \subseteq X$, and for every $g \in F$, there exists an $f \in F$ such that $d\left(f(x), f\left(x_{0}\right)\right)<\varepsilon$ for some $x \in X-A$ and $d(f(a), g(a))<\varepsilon$ for every $a \in A$. Then $F$ is of first category in itself. 
Proof. Let $U$ be an open neighborhood of $x_{0}$ having compact closure. For each $n \in \mathbf{N}$, define

$$
F_{n}=\left\{f \in F \mid d\left(f(x), f\left(x_{0}\right)\right) \geqslant 1 / n \text { for all } x \in X-U\right\} .
$$

To see that $\cup\left\{F_{n} \mid n \in N\right\}=F$, let $f \in F$. Now $f(U)$ is open in $f(X)$, so there exists an open $V$ in $Y$ such that $V \cap f(X)=f(U)$. Also there is an $n \in \mathbf{N}$ such that $B\left(f\left(x_{0}\right), 1 / n\right) \subseteq V$. If $x \in X-U$, then $f(x) \notin f(U)$. But then $f(x) \notin$, $B\left(f\left(x_{0}\right), 1 / n\right)$, so that $f \in F_{n}$.

To see that each $F_{n}$ is closed in $F$, let $n \in \mathbf{N}$ and let $f \in F-F_{n}$. Then there exists an $x \in X-U$ such that $d\left(f(x), f\left(x_{0}\right)\right)<1 / n$. Define $\delta=1 / n-$ $d\left(f(x), f\left(x_{0}\right)\right)$, and define

$$
W=\left[\left[x_{0}, B\left(f\left(x_{0}\right), \delta / 2\right)\right]\right] \cap \cdots \cap[[x, B(f(x), \delta / 2)]],
$$

which is a neighborhood of $f$. If $g \in W$, then

$$
\begin{aligned}
d\left(g(x), g\left(x_{0}\right)\right) & \leqslant d(g(x), f(x))+d\left(f(x), f\left(x_{0}\right)\right)+d\left(f\left(x_{0}\right), g\left(x_{0}\right)\right) \\
& <\delta / 2+d\left(f(x), f\left(x_{0}\right)\right)+\delta / 2=1 / n .
\end{aligned}
$$

Therefore $g \in F-F_{n}$, so that $F_{n}$ is closed.

Finally, to see that each $F-F_{n}$ is dense in $F$, let $n \in \mathbf{N}$ and let $W=\left[\left[A_{1}, V_{1}\right]\right]$ $\cap \cdots \cap\left[\left[A_{k}, V_{k}\right]\right]$ be a basic open subset of $F$ containing some element $g$. There exists some $\varepsilon<1 / n$ such that for each $i=1, \ldots, k, B\left(g\left(A_{i}\right), \varepsilon\right) \subseteq V_{i}$. Define $A=\bar{U} \cup A_{1} \cup \cdots \cup A_{k}$. Then by hypothesis, there exists an $f \in F$ and an $x \in X-A$ such that $d\left(f(x), f\left(x_{0}\right)\right)<\varepsilon$ and $d(f(a), g(a))<\varepsilon$ for all $a \in A$. Therefore $f \in W-F_{n}$, so that $F_{n}$ is nowhere dense in $F$.

EXAMPLE 3. The space $E\left(\mathbf{R}, \mathbf{R}^{2}\right)$ is of first category in itself.

Proof. Let $x_{0}$ be any element of $\mathbf{R}$, let $\varepsilon>0$, let $A$ be a compact subset of $\mathbf{R}$, and let $g \in E\left(\mathbf{R}, \mathbf{R}^{2}\right)$. Let $a<b<c$ be elements of $\mathbf{R}$ such that $[a, c] \subseteq \mathbf{R}-(A \cup$ $\left.\left\{x_{0}\right\}\right)$, and let $y \in B\left(g\left(x_{0}\right), \varepsilon\right)-g(A)$. There exists an arc $\alpha:[a, b] \rightarrow \mathbf{R}^{2}-g(A)$ such that $\alpha(a)=g(a)$ and $\alpha(b)=y$, and there exists an $\operatorname{arc} \beta:[b, c] \rightarrow \mathbf{R}^{2}-(g(A)$ $\cup \alpha([a, b]))$ such that $\beta(b)=y$ and $\beta(c)=g(c)$. Then define $f \in E\left(\mathbf{R}, \mathbf{R}^{2}\right)$ by $f(x)=\alpha(x)$ if $x \in[a, b], f(x)=\beta(x)$ if $x \in[b, c]$, and $f(x)=g(x)$ otherwise. Therefore the property in the Lemma is satisfied, so that $E\left(R, \mathbf{R}^{2}\right)$ is of first category.

EXAMPLE 4. If $\left\{K_{n} \mid n \in \mathbf{N}\right\}$ is a pairwise disjoint family of copies of the Cantor set, then the space $K=\Sigma_{n} K_{n}$ is a hemicompact metrizable space such that $E(K, K)$ and $H(K)$ are both of first category in themselves.

Proof. Let $x_{0}$ be any element of $K$, let $\varepsilon>0$, let $A$ be a compact subset of $K$, and let $g \in E\left(K, K^{2}\right)$ ( $g \in H(K)$, respectively). We may assume that we can find an $x_{1} \in K-A$ such that $g\left(x_{1}\right) \neq g\left(x_{0}\right)$. Let $C_{1}$ be an open subset of $B\left(g\left(x_{0}\right), \varepsilon / 2\right)$ - $\left\{g\left(x_{1}\right)\right\}$ which is a Cantor set, and let $C_{2}$ be an open subset of $K-\left(g(A) \cup C_{1}\right)$ containing $g\left(x_{1}\right)$ which is also a Cantor set. Let $\alpha$ be a homeomorphism from $C_{1} \cup C_{2}$ onto $C_{1} \cup C_{2}$ such that $\left(C_{1} \cup\left\{x_{1}\right\}\right) \subseteq C_{1}$. Now let $h \in H(K)$ be defined by $h(x)=\alpha(x)$ if $x \in C_{1} \cup C_{2}$ and $h(x)=x$ otherwise. Then define $f$ by $f=$ $h \circ g$, which is an element of $E(K, K)$ (an element of $H(K)$, respectively). The conclusion now follows from the Lemma. 
We will give one more example showing that $E(X, X)$ and $H(X)$ may have different properties. It will be useful to have the following fact to use on this example.

Proposition. If $\left\{X_{n} \mid n \in \mathbf{N}\right\}$ is a family of pairwise disjoint copies of some connected space, then $H\left(\Sigma_{n} X_{n}\right)$ is homeomorphic to $\mathbf{P} \times \Pi_{n} H\left(X_{n}\right)$, where $\mathbf{P}$ is the space of irrationals.

Proof. For each $m, n \in \mathrm{N}$, let $\alpha_{m}^{n}: X_{m} \rightarrow X_{n}$ be a homeomorphism. Define

$$
\Phi: H\left(\sum_{n} X_{n}\right) \rightarrow H(\mathrm{~N}) \times \prod_{n} H\left(X_{n}\right)
$$

as follows. Let $h \in \Sigma_{n} X_{n}$. Then take

$$
\Phi(h)=\left\langle\phi,\left\langle\alpha_{\phi(n)}^{n} \circ\left(\left.h\right|_{X_{n}}\right)\right\rangle_{n}\right\rangle
$$

where $\phi \in H(\mathrm{~N})$ is defined by: $\phi(n)$ is that element of $\mathrm{N}$ such that $h\left(X_{n}\right)=X_{\phi(n)}$. It is not difficult to check that $\Phi$ is a bijection.

Let $\pi: H(\mathrm{~N}) \times \Pi_{n} H\left(X_{n}\right) \rightarrow H(\mathrm{~N})$ and, for each $m \in \mathrm{N}$, let $\pi_{m}: H(\mathrm{~N}) \times$ $\Pi_{n} H\left(X_{n}\right) \rightarrow H\left(X_{m}\right)$ be the projection maps. Now $\pi \circ \phi$ is continuous since for each $n \in \mathrm{N}, \phi \in H(\mathrm{~N})$, and $x \in X_{n}$, it is the case that $(\pi \circ \phi)\left(\left[\left[x, X_{\phi(n)}\right]\right]\right) \subseteq$ [[ $\{n\},\{\phi(n)\}]]$. Also each $\pi_{m}{ }^{\circ} \phi$ is continuous since for each compact $A \subseteq X_{m}$, each open $W \subseteq X_{m}$, and each $\phi \in H(\mathrm{~N})$, we have $\left(\pi_{m} \circ \phi\right)\left(\left[\left[A,\left(\alpha_{\phi(m)}^{m}\right)^{-1}(W)\right]\right] \subseteq\right.$ [[ $A, W]]$. Therefore $\Phi$ is continuous.

To see that $\Phi^{-1}$ is continuous let $[[A, W]]$ be a subbasic open set in $H\left(\Sigma_{n} X_{n}\right)$ and let $\phi \in H(\mathbf{N})$. There is some $N \in \mathbf{N}$ such that $A \subseteq X_{1} \cup \cdots \cup X_{n}$. For each $n=1, \ldots, N$, let $A_{n}=A \cap X_{n}$ and $W_{n}=W \cap X_{\phi(n)}$. Then

$$
\begin{aligned}
\pi^{-1}([[\{1\},\{\phi(1)\}]] \cap \cdots \cap[[\{N\},\{\phi(n)\}]]) \\
\cap \pi_{1}^{-1}\left(\left[\left[A_{1}, W_{1}\right]\right]\right) \cap \cdots \cap \pi_{N}^{-1}\left(\left[\left[A_{N}, W_{n}\right]\right]\right) \subseteq([[A, W]]),
\end{aligned}
$$

so that $\Phi^{-1}$ is continuous.

Now we show that $H(\mathbf{N})$ is a $G_{\delta}$-set in $E(\mathbf{N}, \mathbf{N})=I(\mathbf{N}, \mathbf{N})$, which is completely metrizable. First note that for each $n, F_{n} \equiv[[\mathbf{N}, \mathbf{N}-\{n\}]]$ is a closed subset of $E(\mathbf{N}, \mathbf{N})$ contained in $E(\mathbf{N}, \mathbf{N})-H(\mathbf{N})$. If $f \in E(\mathbf{N}, \mathbf{N})-H(\mathbf{N})$, then there exists an $n$ such that $f \in F_{n}$. Therefore $E(\mathbf{N}, \mathbf{N})-H(\mathbf{N})=\cup\left\{F_{n} \mid n \in \mathbf{N}\right\}$, so that $H(\mathbf{N})$ is a $G_{\delta}$-set in $E(\mathbf{N}, \mathbf{N})$. Finally, $H(\mathbf{N})$ is homeomorphic to $\mathbf{P}$ since every separable zero-dimensional completely metrizable space with no nonempty compact open subset is homeomorphic to $\mathbf{P}$ (see [1]).

EXAMPLE 5. If

$$
Z=\{0\} \cup\left(\cup\left\{(n, n+1) \cup\left[1 / 2^{2 n}, 1 / 2^{2 n-1}\right] \subseteq \mathbf{R} / n \in \mathbf{N}\right\}\right)
$$

with the usual topology, then $Z$ is a hemicompact metric space such that $E(Z, Z)$ is of first category in itself while $H(Z)$ is completely metrizable.

Proof. For each $n$, let $R_{n}=(n, n+1)$, let $R=\cup\left\{R_{n} \mid n \in \mathrm{N}\right\}$, and let $C=Z$ $-R$. From the Proposition it follows that $H(R)$ is homeomorphic to $\mathbf{P} \times$ $\Pi_{n} H\left(R_{n}\right)$, so that $H(Z)$ is homeomorphic to $H(C) \times \mathrm{P} \times \Pi_{n} H\left(R_{n}\right)$, which is completely metrizable. On the other hand, it follows from the Lemma that $E(Z, Z)$ 
is of first category. To see this, let $\varepsilon>0$, let $A$ be a compact subset of $Z$, and let $g \in E(Z, Z)$. For each $n$, define

$$
A_{n}=\{0\} \cup\left(\cup\left\{\left[1 / 2^{2 i}, 1 / 2^{2 i-1}\right] \mid i \geqslant n\right\}\right) .
$$

Now there is some $n$ such that $1 / 2^{2 n-1}<\varepsilon, R_{n} \cap g(A)=\varnothing$, and $R_{n} \cap g(Z) \neq \varnothing$. Let $\alpha$ be a homeomorphism from $A_{n}$ onto $A_{n+1}$, and let $\beta$ be an embedding of $R_{n}$ into $\left[1 / 2^{2 n}, 1 / 2^{2 n-1}\right]$. Let $h \in E(Z, Z)$ be defined by $h(z)=\alpha(z)$ if $z \in A_{n}$, $h(z)=\beta(z)$ if $z \in R_{n}$, and $h(z)=z$ otherwise. Finally take $f=h \circ g$.

These examples suggest the following questions for a hemicompact metric space $X$. If $E(X, X)$ is completely metrizable, is $H(Z)$ completely metrizable? If $H(X)$ is of second category in itself, must it be completely metrizable? Is there a convenient characterization for $H(X)$ being completely metrizable?

\section{REFERENCES}

1. P. Alexandroff and P. Urysohn, Über nulldimensionale Punktmengen, Math. Ann. 98 (1928), 89-106.

2. R. F. Arens, A topology for spaces of transformations, Ann. of Math. 47 (1946), 480-495.

3. E. Beckenstein, L. Narici and C. Suffel, Topological algebras, Notas de Matematica, No. 60, North-Holland, New York, 1977.

4. C. Kuratowski, Evaluation de la classe borèlienne ou projective dín ensemble de points à l'aide des symboles logiques, Fund. Math. 17 (1931), 249-272.

Department of Mathematics, Virginia Polytechic Institute and State University, BlacksBURG, VIRGINIA 24061 